\title{
Vitamin B12 Deficiency Presenting as Acute Psychosis
}

\author{
Manoj Kumar Sahoo¹, Anupama Sahu², Harshita Biswas³, Sanjay Kumar Agarwal4
}

${ }^{1}$ Department of Psychiatry, Tata Main Hospital, Jamshedpur, Jharkhand, India. ${ }^{2}$ Department of Medicine, Tata Main Hospital, Jamshedpur, Jharkhand, India. ${ }^{3}$ Department of Psychiatry, Tata Main Hospital, Jamshedpur, Jharkhand, India.

${ }^{4}$ Department of Psychiatry, Tata Main Hospital, Jamshedpur, Jharkhand, India.

\section{INTRODUCTION}

Psychiatric symptoms attributable to vitamin B12 deficiency have been described for decades. Vitamin B12 deficiency is common in developing countries and its prevalence ranges up to $67 \%$ among Indian population. ${ }^{1}$ The earlier reports are for the most part in accordance with more recent ones, despite being diagnostically less specific in psychiatric and haematological terms. These symptoms seem to fall into several clinically separate categories like slow cerebration, confusion, memory changes, delirium, with or without hallucinations and/or delusions, depression, acute psychotic states, and (more rarely) reversible manic and schizophreniform states. ${ }^{2}$ Apart from these conditions, some cases of dementia, violent behaviour and fatigue have also been documented to be caused by vitamin B12 deficiency. ${ }^{3}$ Reports of patients initially presenting to psychiatric facilities without associated haematological manifestations are rare. There are a few case reports of acute psychosis attributed to vitamin B12 deficiency, but more evidence is required to substantiate and establish this association. ${ }^{4}$ We describe three patients who developed acute psychosis accompanied by features suggestive of organic aetiology. They were found to have cobalamin deficiency in the absence of typical neurological or haematological abnormalities.

B12 deficiency is widely prevalent and usually presents with hematologic and neuropsychiatric manifestations. Psychiatric symptoms seldom precede anaemia and present as the principal manifestation of B12 deficiency. Reports of patients initially presenting to psychiatric facilities without associated haematological manifestations are rare. We report 3 cases of vitamin B12 deficiency who developed symptoms of acute psychosis in the absence of neurological symptoms or typical haematological abnormalities. Three of them improved with Intramuscular Methylcobalamin and low dose antipsychotics. They remained asymptomatic at 1 year of follow up after stoppage of antipsychotics. All the three patients were advised dietary modifications, supplementation of Vitamin B 12 and periodic monitoring of Vitamin B12 level to prevent relapse.

\author{
Corresponding Author: \\ Dr. Manoj Kumar Sahoo, \\ OPD Complex Room-107, \\ Tata Main Hospital, \\ Bistupur, Jamshedpur-831001, \\ Jharkhand, India. \\ E-mail:drmanojsahoo@gmail.com
}

DOI: $10.14260 / \mathrm{jemds} / 2020 / 105$

Financial or Other Competing Interests: None.

How to Cite This Article:

Sahoo MK, Sahu A, Biswas H, et al. Vitamin B12 deficiency presenting as acute psychosis. J. Evolution Med. Dent. Sci. 2020;9(07):465-467, DOI:
Submission 13-12-2019,

Peer Review 22-01-2020,

Acceptance 29-01-2020,

Published 17-02-2020. 


\section{Case 1}

A 31-year-old married female, presented with Complaints of behavioural changes, paranoid delusions, insomnia \& agitation. The onset was acute over a period of 4-5 days. On mental status examination, she had persecutory delusions, lack of insight \& normal cognitive functions. On physical examination, there were no neurological deficits \& examination of other systems was normal. Patient's dietary habits were vegetarian and there was no history of any substance abuse.

\section{Case 2}

A 54 year married male, presented with Complaints of overtalkativeness, irrelevant talk, agitation, paranoid ideation and inappropriate laughter for 3-4 days. He was a known case of hyperthyroidism. During the time of admission his thyroid function was normal, and he was on regular medication (neomercazole). On mental status examination, he was overtalkative, restless and voicing paranoid thoughts about his neighbour and colleagues. His cognitive functions were normal. On physical examination there were no neurological deficits \& other systems were normal. Patient's dietary habits were Vegetarian and no history of any substance abuse.

\section{Case 3}

A 34-year unmarried female, presented with complaints of abnormal behaviour, irrelevant talk and talking to self, for generalised weakness for 3-4 weeks. On mental Status examination: She had auditory hallucinations, delusion of persecution, apathy and shallow affect. On physical examination she had pallor (dimorphic anaemia with both microblasts and megaloblasts on peripheral smear). There were no neurological deficits. Patient's dietary habits were Vegetarian and no history of any substance abuse. She was further investigated for her raised bilirubin level, her ultrasound abdomen and liver enzymes were normal. After she received 2 units of packed cell blood transfusion and Vitamin B12 injections, her bilirubin level came down to 0.45 and remained within normal limits in follow-up. As her investigations of other systems were normal, polyglandular autoimmune diseases was not considered. The indirect bilirubin level may be elevated because of haemolysis associated with vitamin B12 deficiency. ${ }^{5}$

\begin{tabular}{|c|c|c|c|c|c|c|}
\hline & & Range & UOM & Case 1 & Case 2 & Case 3 \\
\hline \multirow{2}{*}{$\mathrm{CBC}$} & $\mathrm{HB}$ & $11.5-16.5$ & $\mathrm{gm} / \mathrm{dl}$ & 12.0 & 11.7 & 5.7 \\
\hline & MCV & $76-96$ & fl & 87.6 & 85.5 & 115.3 \\
\hline TSH & & 0.4 to 5.0 & $\mathrm{mIU} / \mathrm{L}$ & 5.09 & 4.5 & 5.43 \\
\hline VitB12 & & $180-900$ & $\mathrm{ng} / \mathrm{mL}$ & 27.6 & 85 & 54 \\
\hline Folate & & $2-20$ & $\mathrm{ng} / \mathrm{mL}$ & 7.18 & 5.83 & 5.55 \\
\hline Bone Marrow & & & & Notdone & Notdone & dimorphic anaemia \\
\hline Neuro imaging & & & & Normal & Normal & Normal \\
\hline UGIE & & & & Notdone & Notdone & $\begin{array}{c}\text { Mild diffused gastritis } \\
\text { with normal gastric } \\
\text { biopsy }\end{array}$ \\
\hline \multirow{2}{*}{ LFT } & Bilirubin & $0.2-1.2$ & $\mathrm{mg} / \mathrm{dL}$ & 1.1 & 0.44 & $2.19(0.98 / 1.21)$ \\
\hline & Liver enzymes & & & Normal & Normal & Normal \\
\hline \multicolumn{7}{|c|}{ Investigations } \\
\hline
\end{tabular}

\section{Treatment History}

All the three patients were treated with low dose antipsychotics. Case 1 was given olanzapine $5 \mathrm{mg}$ and case 2 \& 3 were treated with Risperidone up to $2 \mathrm{mg}$ along with Intramuscular injection of Methylcobalamin $500 \mathrm{mg}$ daily for the hospitalised period followed by once in a month till 6 months and then shifted to tab Methylcobalamin with periodical monitoring of vitamin B12 level. Antipsychotics were stopped after 1 month in case 1 and 2 and after 3 months in case 3.

\section{DISCUSSION}

Vitamin B12 is one of the most essential vitamins affecting various systems of the body. Cases of neuropsychiatry disorders due to its deficiency are more common in elderly patients with prevalence of $10-20 \%$. The most common psychiatry symptoms reported in the literature associated with vitamin B12 deficiency was depression, mania, psychotic symptoms, cognitive impairment and delirium. ${ }^{6}$ Mental disturbances due to vitamin B12 deficiency have been reported previously as the chief or sole symptom,? occasionally even preceding the decrease of cobalamin in the serum. Psychosis related to vitamin B12 deficiency, however, is considered very rare and has received little attention in the literature. A possible mechanism for psychotic behaviour was suggested by Hutto, ${ }^{8}$ who reported that cobalamin and folate participate in the synthesis of monoamine neurotransmitters in the brain by increasing $\mathrm{BH} 4$ (tetrahydrobiopterin) synthesis. All patients presented with acute onset psychotic symptoms with significant socio occupational dysfunctions. The detailed interview with the 3 patients revealed acute psychoses with no personal or family history of psychiatric illness and substance abuse. On detail exploration we didn't find any significant stressors contributing to such behaviour. Therefore, we went for detail investigations to find out possible organic aetiology for above symptoms.

On investigation, all 3 of them had significant low level of serum vitamin B12 which can be because of the vegetarian dietary habits. Their dietary analysis showed that their daily diet was based predominantly on pulses, rice and vegetables, with a minimal intake of dairy products, which are the sole dietary sources of vitamin B12. Such people are at a higher risk, as they don't consume the major food sources of B12: meat, eggs and dairy products. Diagnosis of vitamin B12 deficiency is typically based on measurement of serum vitamin B12 levels; however, about 50 percent of patients with subclinical disease have normal B12 levels. A more sensitive method of screening for vitamin B12 deficiency is measurement of serum methylmalonic acid and homocysteine levels, which are increased early in vitamin B12 deficiency. Since all the three patients had significant low level of vitamin B12, we did not consider the measurement of homo cysteine levels, MMA level. ${ }^{9}$ Normal haemoglobin, haematocrit and mean corpuscular volume (MCV) value in two of the patients and normal UGIE in third patient rules out possibility of pernicious anaemia. Only factors which could have contributed for the symptoms may be significant stress but in the present cases no stressor was present. Such low level of vitamin B12, with good response to combination of Methylcobalamin and low dose of antipsychotics and no relapse of symptoms after stoppage of antipsychotics indicate vitamin B12 deficiency induced acute psychosis.

This case series reports rare cases of vitamin B12 deficiency induced psychosis. These cases suggest that B12 
deficiency can present as an isolated acute psychotic episode. It underlines the importance of evaluation of vitamin B12 and other potential reversible causes of psychosis. We therefore recommend that vitamin B12 levels be determined in patients with mental and psychiatric disturbances to prevent severe and irreversible complications.

\section{CONCLUSIONS}

Though there are several case reports of vitamin B12 deficiency and various neuropsychiatric symptoms, isolated acute psychosis without haematological and neurological abnormality is rare. So, it is advisable to screen patients with vegetarian diet \& acute behavioural disturbances for Vitamin B12 deficiency even in absence of haematological and neurological symptoms.

\section{REFERENCES}

[1] Yajnik CS, Deshpande SS, Lubree HG, et al. Vitamin B12 deficiency and hyperhomocysteinemia in rural and urban Indians. J Assoc Physicians India 2006;54:775-82.
[2] Dommisse J. Subtle vitamin deficiency and psychiatry: a largely unnoticed and devastating relationship. Med Hypothesis 1991;34 (2):131-40.

[3] Hector M, Burton JR. What are the psychiatric manifestations of vitamin B12 deficiency? J Am Geriatr Soc 1988;36 (12):1105-12.

[4] Berry N, Sagar R, Tripathi BM. Catatonia and other psychiatric manifestation of vitamin $B_{12}$ deficiency and related issues. Acta Psychiatr Scand 2003;108:156-9.

[5] Waheed MA, Elzouki A. Vitamin $B_{12}$ deficiency presenting as haemolytic anemia. Libyan J Med Sci 2018;2 (3):114-5.

[6] Carvalho AR, Vacas S, Klut C. Vitamin $B_{12}$ deficiency induced psychosis - a case report. European Psychiatry 2017; (supple 41):S805.

[7] Payinda G, Hansen T. Vitamin $B_{12}$ deficiency manifested as psychosis without anemia [Letter]. Am J Psychiatry 2000;157 (4):660-1.

[8] Hutto BR. Folate and Cobalamin in psychiatric illness. Compr Psychiatry 1997;38 (6):305-14.

[9] Robert C, David LB. Vitamin $B_{12}$ deficiency. Am Fam Physician 2003;67 (5):979-86. 